\title{
Mach-Zehnder interferometric filter based planar Doppler velocimetry (MZI-PDV)
}

\author{
Zenghai H. Lu ${ }^{1}$, Tom. O.H. Charrett ${ }^{2}$, Helen D. Ford ${ }^{3}$, Ralph P. Tatam ${ }^{4}$
}

\author{
1: Engineering Photonics Group, Cranfield University, UK, z.h.lu@cranfield.ac.uk \\ 2: Engineering Photonics Group, Cranfield University, UK, t.charrett@cranfield.ac.uk \\ 3: Engineering Photonics Group, Cranfield University, UK, h.d.ford@cranfield.ac.uk \\ 4: Engineering Photonics Group, Cranfield University, UK, r.p.tatam@cranfield.ac.uk
}

\begin{abstract}
This paper describes the development of a Mach-Zehnder interferometric filter based planar Doppler velocimetry (MZI-PDV) flow measurement technique. The technique uses an unbalanced MachZehnder interferometer (MZI) to convert Doppler frequency shifts into intensity variations. The free spectral range of the interferometric filter can be selected by adjusting the optical path difference of the MZI. This allows the velocity measurement range and resolution to be varied. In contrast to molecular filter based PDV any laser source with single-frequency operation and a narrow linewidth can be used as the requirement for a suitable absorption line is no longer necessary.

The processing methods used to extract the velocity information are described and discussed. The construction of a MZI-PDV system that incorporates a phase-locking system designed to stabilize the filter is described and example measurements made on the velocity field of a rotating disc and an axis-symmetric air jet are presented.
\end{abstract}

\section{Introduction}

Knowledge of fluid velocities is essential in a variety of engineering applications, both in external flows e.g. wind tunnel measurements for vehicle prototypes in the aerospace and automotive industries, and in internal turbo machinery flows. Planar Doppler velocimetry (PDV)[1,2], also known as Doppler Global Velocimetry (DGV)[3,4] allows the measurement of the velocity over a plane defined by a laser light sheet by measuring the Doppler frequency shift of light scattered from particles in the flow.

$$
\Delta v=\frac{v_{0}(\hat{o}-\hat{i}) \cdot \mathrm{V}}{c}
$$

Where $\Delta v$ is the Doppler frequency shift of the scattered light, $v_{0}$ is the illumination frequency and $\mathrm{V}$ is the velocity vector associated with the scattering particles, $c$ is the free space speed of light and $\hat{o}$ and $\hat{\imath}$ are unit vectors in the observation and illumination directions respectively.

This is done using an absorption line of molecular iodine as a frequency-to-intensity filter. By selecting the illumination frequency to coincide with an absorption line of the gas, the received intensity at the CCD camera is directly related to the Doppler shift, and hence the flow velocity. A reference camera, viewing the flow directly, is needed to normalize the signal for variations in scattered light intensity due to effects other than the flow velocity.

However, the use of molecular gas filters has several disadvantages. The first is that the choice of lasers is limited by the requirement to be able to tune to an appropriate absorption line. The second is that the transfer function is determined by the form of the gas absorption line and therefore the sensitivity can not be varied greatly, although it is possible to change the shape of the spectral feature by varying the concentration of iodine, the cell temperature or by the addition of buffer gases to broaden the absorption line. 
In this work the possibility of replacing the iodine cell with a Mach-Zehnder interferometric filter, as the frequency-to-intensity transducer in a PDV system has been investigated. The use of a MachZehnder interferometer has several advantages over molecular filters, the choice of illumination laser is no longer limited by the availability of a suitable absorption line and the path length imbalance of the interferometer can be selected to correspond to the expected velocity range for a given situation. Michelson interferometers have previously been used to make planar single component velocity measurements [5,6]. However the Mach-Zehnder interferometer offers the advantage of providing two complementary outputs allowing the scattered light intensity to be normalized by taking the difference of the two outputs divided by the sum potentially increasing the sensitivity of the system. A system capable of making either single velocity component measurements [7], or three component velocity measurements by incorporating imaging fibre bundles [2], was constructed and demonstrated by making measurements of the velocity of a rotating disc and a seeded air jet.

\section{Mach-Zehnder Interferometric filter}

Figure 1 shows the arrangement of an unbalanced Mach-Zehnder interferometer (MZI) for PDV. A laser light sheet, of frequency $v_{0}$, illuminates the object plane $\Sigma$ located in the flow. Light scattered from particles seeded in the flow is collected and coupled into the interferometer. Frequency variations caused by the Doppler shift are converted into variations in the recorded intensities on CCD detectors located at $\Sigma$ ', and $\Sigma$ '. The scattered light coming from the object plane $\Sigma$ is collimated by lens L1 and then divided by BS1 (beam splitter, 50:50 split ratio) into two beams of equal intensity, which follow paths 11 and 12 . The light in path 11 is turned at mirror M2 and prism 2 before being split by BS2 and imaged onto the two detectors located at $\Sigma$ ', and $\Sigma$ ' using lenses L2 and L3. The second path, 12 passes through a glass block G, with a length 1 and refractive index $\mathrm{n}$. The light is retro-reflected by prism 1, making a second pass through the glass block. It is then turned at mirror, M1 and recombined with the light in the first path at BS2.

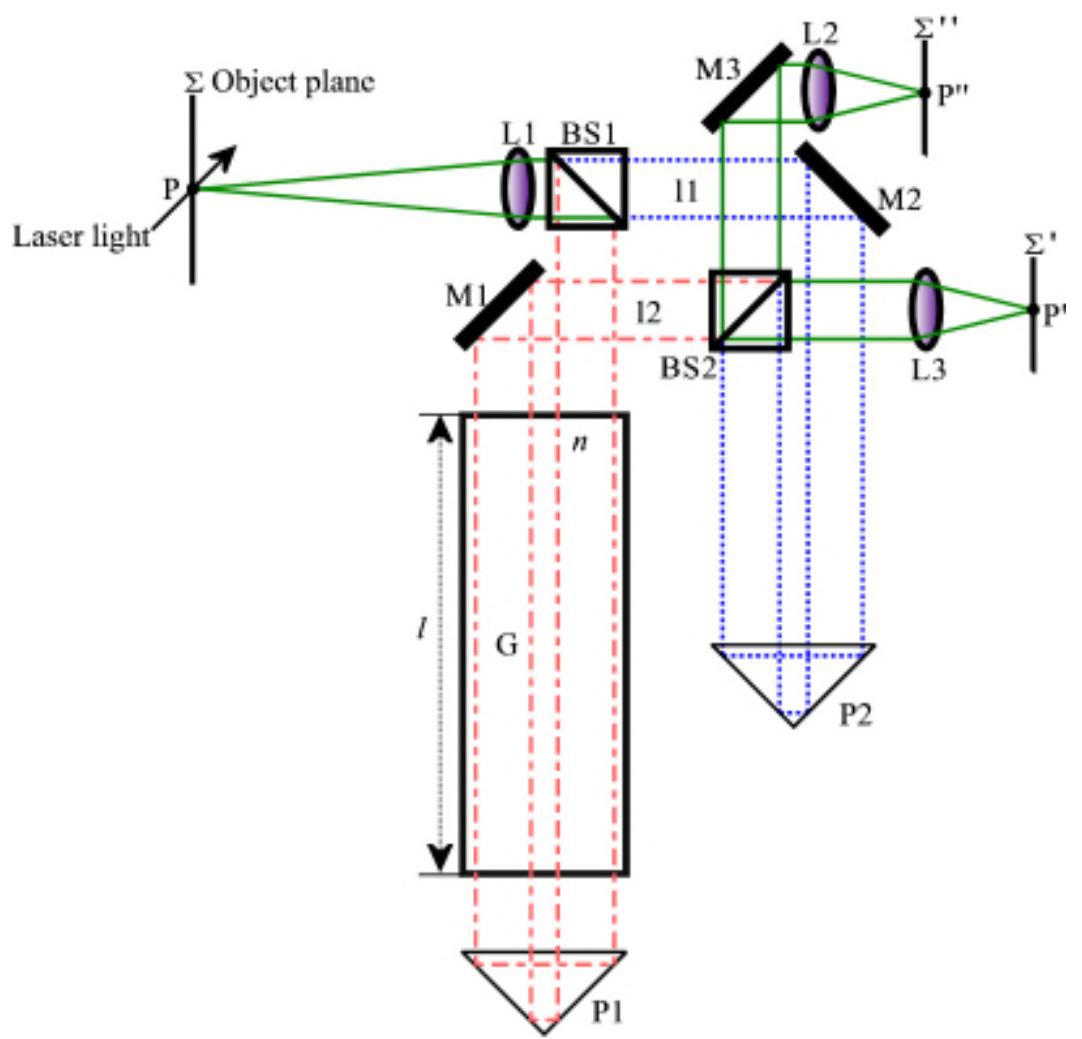

Fig. 1 An unbalanced Mach-Zehnder interferometer. The beam paths are shown in different styles: path 11 is dashed, 12 is dot-dashed and the combined path is solid 
For a MZI, the intensity at the two outputs of the interferometer will depend on the phase difference, $\Delta \varphi$, between the two arms.

$$
\begin{aligned}
& I_{1} \propto I_{0} \cos ^{2}\left(\frac{\Delta \varphi}{2}\right) \\
& I_{2} \propto I_{0} \sin ^{2}\left(\frac{\Delta \varphi}{2}\right)
\end{aligned}
$$

The normalized intensity, $I_{N}$, can be expressed as:

$$
I_{N}=\frac{I_{1}-I_{2}}{I_{1}+I_{2}} \propto V \cdot \cos (\Delta \varphi)
$$

where $V$ is the interference fringe visibility determined by the transmission performance of the whole optical set-up. The normalized intensity, $I_{N}$, is a function only of the phase difference $\Delta \varphi$ and is independent of the scattered light intensity. The phase difference $\Delta \varphi$ is a function of the optical path difference $(\Delta l)$ between the two arms, the light frequency $(v)$ and the speed of light $(c)$ :

$$
\Delta \varphi=\frac{2 \pi \Delta l}{c} v
$$

Therefore, any Doppler shift of the scattered light will cause a variation of the normalized intensity. The optical path difference can be varied by selecting the length $l$ of the glass block allowing the velocity measurement range and resolution to be varied by selecting a suitable path length imbalance and glass block. The primary purpose of the glass block is to correct for the difference in magnification and focal distance in the two arms of the interferometer. For example a $5 \mathrm{~cm}$ BK7 glass block (refractive index, $\mathrm{n}=1.52$ ) has a free-spectral-range (FSR) of about $7 \mathrm{GHz}$ at $514.5 \mathrm{~nm}$, it this was increased to $50 \mathrm{~cm}$ then FRS is reduced to $0.7 \mathrm{GHz}$. The technique is therefore limited for low velocities due to the long block length required, which leads to optical distortions, phase instabilities and vignetting effects in the images. These effects are greater for larger optical path differences.
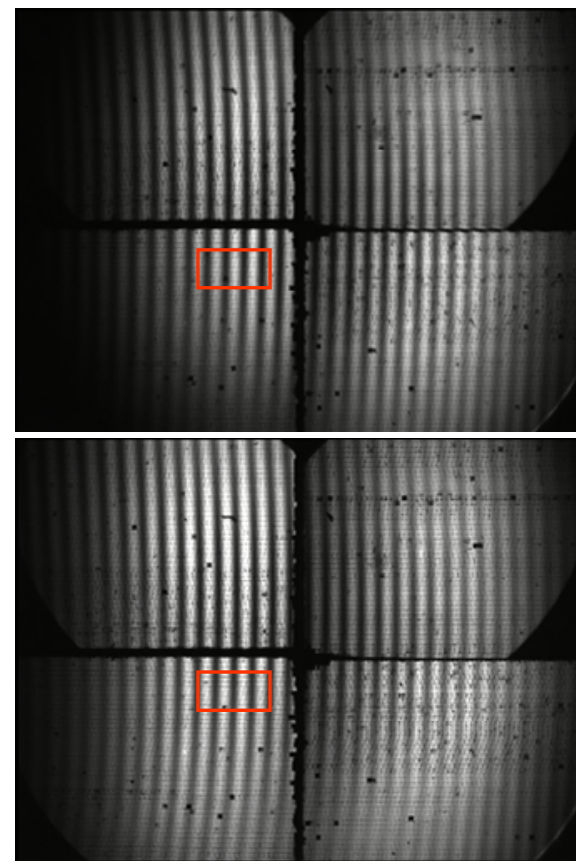

(a)

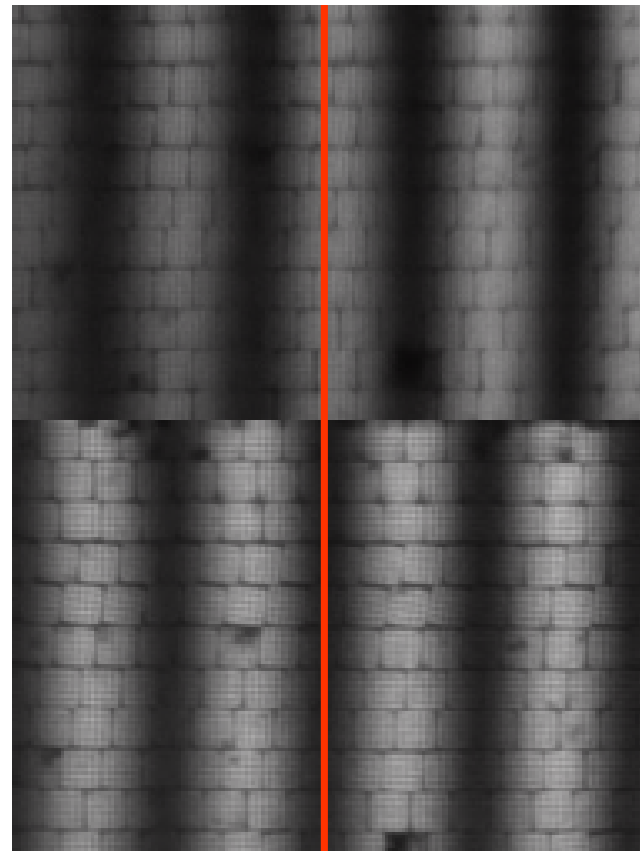

(b)

Fig. 2 (a) Example images captured by CCD1 (top) and CCD2(bottom), showing a rotating disc illuminated with the argon ion laser at $514.5 \mathrm{~nm}$ and viewed using the imaging fibre bundles. (b) Magnified section highlighted in (a) showing the fringes in anti-phase and the bundle structure. 
Examples of typical images captured at the two outputs are shown in figure 2(a). Fringes are formed in the images due to a varying phase difference across the field of view, the resulting fringes are concentric rings centred about the axis of the interferometer. However due to difficulties in accurately aligning the optical components the fringes will actually appear as curved fringes show in figure 2, and the number of fringes across the field of view can be adjusted by introducing additional tilt into one arm of interferometer. The fringes at the two outputs of the interferometer will be in anti-phase as can be seen in the magnified region shown in figure 2(b).

\section{Experimental Configuration}

A schematic of the experimental arrangement used is shown in figure 3 . The illumination laser was a continuous wave argon ion laser, allowing time averaged velocity measurements to be made. Light from the laser was coupled into an optical fibre through a Faraday isolator, and the output was formed into a light sheet using a prism-scanning device that scanned the beam rapidly across the measurement area. The scattered light from a flow region can be imaged either using a single SLR camera lens or using multiple lenses for multiple component measurements using the imaging fibre bundle approach previously reported [2].

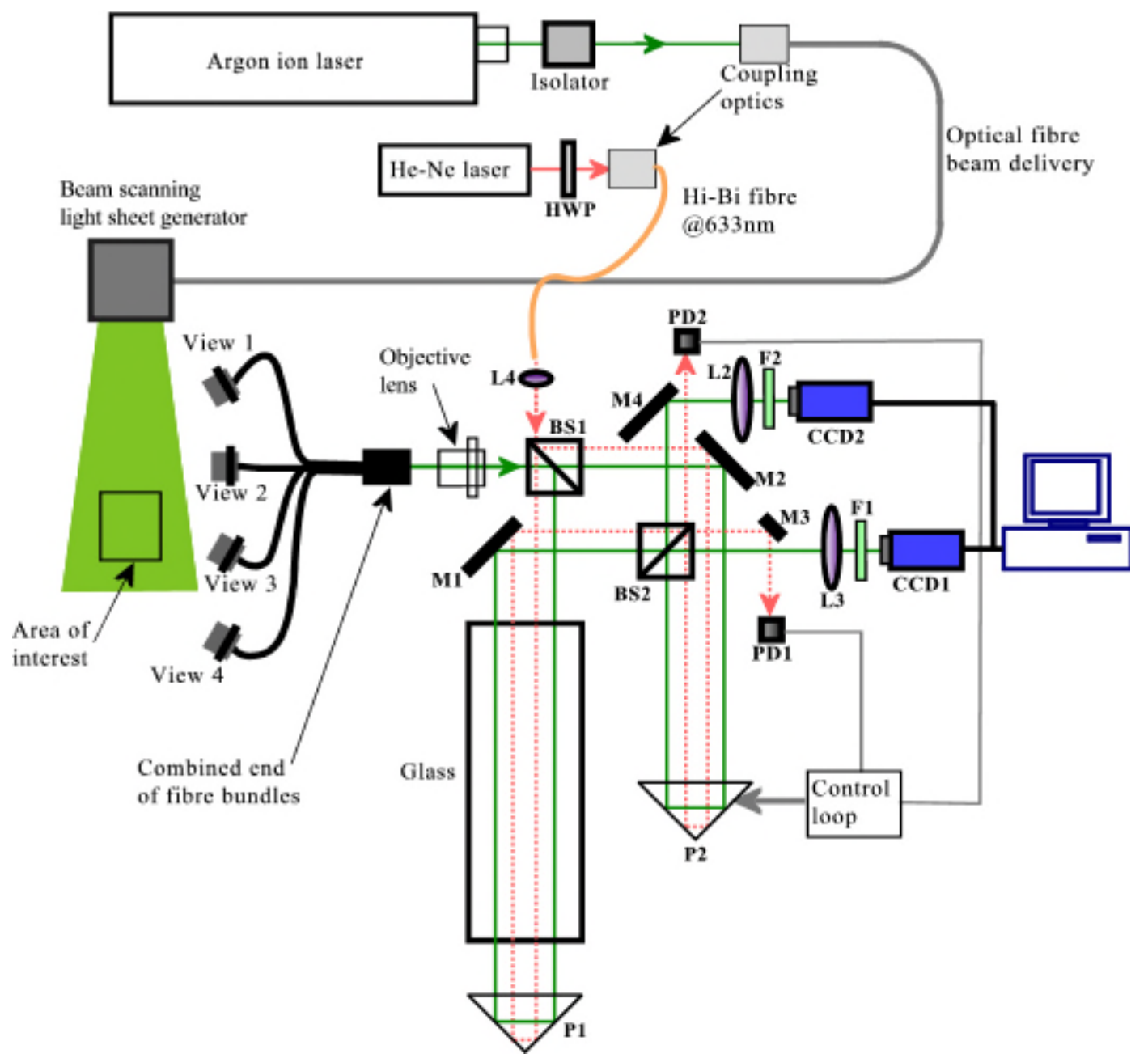

Fig. 3 The experimental arrangement; including detail of the phase locking system to stabilise the MZI.

HWP: half-wave plate; BS1, 2: non-polarisation beam splitters; L2, 3: tube lenses; L4: coupling lens; M1, 2, 3, 4: mirrors; F1, 2: green filters; P1, 2: right-angle prisms. Beam path for measurement system shown as solid line, beam path for phase locking system shown as a dotted line. 
The imaging fibre bundles used here are coherent array of fibres that is split into four channels. Each channel comprises $600 \times 500$ fibres, $8 \mu \mathrm{m}$ in diameter and positioned on $10 \mu \mathrm{m}$ centres. The bundle constructed by stacking $25 \times 25$ pixel multi-fibre blocks, these can be seen in figure $2(\mathrm{~b})$. The MZI was constructed using an infinity-corrected microscope optical system consisting of a matched objective and a tube lens. The two complementary output images of the MZI were captured by the two CCD cameras. In this work, a $15 \mathrm{~cm}$ BK7 glass block was used giving a FSR of around $1.16 \mathrm{GHz}$ and a velocity range of $\sim \pm 150 \mathrm{~m} / \mathrm{s}$. This is similar to the width of the iodine absorption line (in the order of $1 \mathrm{GHz}$ ) used in conventional PDV systems and allows comparison with results from PDV systems.

In order to prevent the interferometer phase drift due to temperature variations and mechanical vibrations, a phase locking system was designed using a feedback loop controller to adjust the path difference. This uses a $633 \mathrm{~nm}$ beam from a single-frequency stabilised He-Ne laser directed through the interferometer near the periphery of the image area. Two photodiodes (PD1 \& PD2) were used to monitor the complementary interferometric outputs. Two band pass filters (F1 \& F2) with a transmission band of $400-580 \mathrm{~nm}$ and $>85 \%$ transmission prevented light from the He-Ne locking laser being detected by the CCD cameras. The signal from the photodiodes were used with a custom designed PID electronic circuit that generated a error signal that was fed back onto the piezoelectrically controlled stage supporting the right angle prism, P2, to modify the interferometer path difference. The operational stability depends mainly on the frequency stability of the He- $\mathrm{Ne}$ laser $\left( \pm 2 \mathrm{MHz}\right.$ in 1 hour plus $\left.0.5 \mathrm{MHz} /{ }^{\circ} \mathrm{C}\right)$, corresponding to $\sim \pm 1 \mathrm{~m} / \mathrm{s}$ velocity error.

\section{Processing methods}

An overview of the processing used is shown in Figure 4. The key stage in the processing is the calculation of the phase shift between the flow on and flow off images. Several methods of calculating the phase shift have been investigated; the first uses the shift of the fringe pattern minima and is described in section Error! Reference source not found.. This method only allows the Doppler shift to be found at positions that correspond with minima in the fringe images. The second and third methods allow a measurement to be made at every pixel. Method two, that was previously reported [7] is the calculation of the phase at every pixel using the normalised intensity and is described in section 4.2. A third method is also presented here that uses Fourier domain processing to evaluate the phase and is described in section 4.3. All the methods described here require two pairs of images to be captured; one pair of images is captured under zero-velocity conditions ( $\mathrm{I} 1_{0}$ and $\left.\mathrm{I} 2_{0}\right)$ and the second pair with the flow on (I1 and I2).

The Doppler shift responsible for a given phase shift can then be found using equation (6):

$$
\Delta v=\frac{c}{\Delta l} \frac{\left(\Delta \varphi-\Delta \varphi_{0}\right)}{2 \pi}
$$

Once the Doppler shift has been calculated, the views are spilt into separate images and de-warped to a common grid (if using the bundles for multiple component measurements). Each velocity component is then calculated using the Doppler equation (1) using the observation and illumination vectors calculated for that view. The final stage is to transform from the non-orthogonal measured velocity components to the orthogonal velocity components aligned with the experimental axis. The use of the imaging fibre bundles allows an extra velocity component to be measured in addition to the three required for this transformation. The use of the extra components in the calculation can reduce the propagation of errors in this process by providing redundant data [9]. 


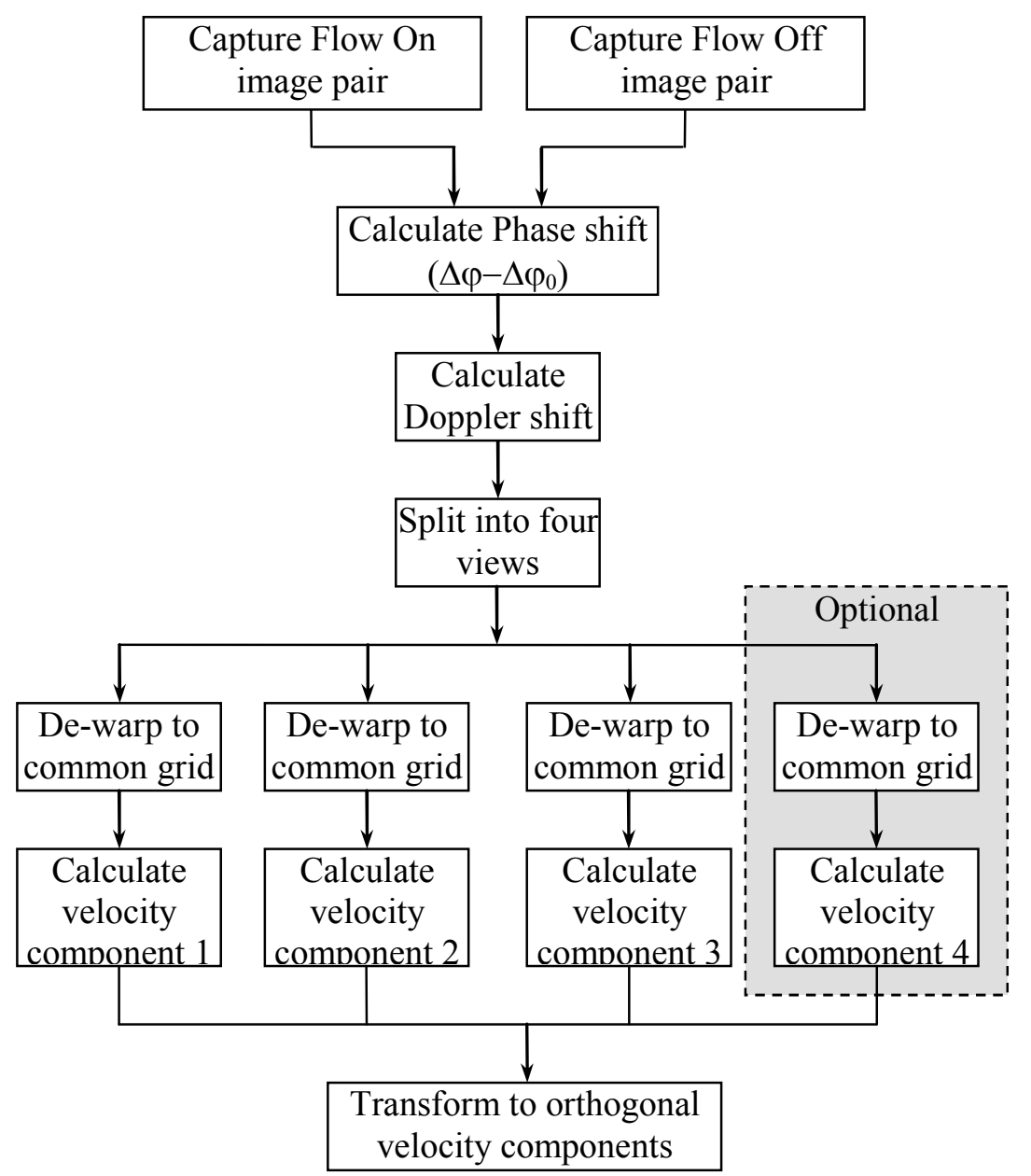

Fig. 4 General processing scheme used to calculate the three component velocity using a MZI-PDV system.

\subsection{Velocity calculation based on the shift of the fringe pattern}

The output light intensity of a MZI depends upon its optical phase and the original light intensity distribution and will have a co-sinusoidal form as given by equations (2) and (3). At $\Delta \varphi=\pi, 3 \pi, \ldots$ for the first output (CCD1) and $\Delta \varphi=0,2 \pi, \ldots$ for the second output (CCD2) the intensity will be at a minimum and equidistant minima are present in the recorded images. When the flow velocity causes a Doppler shift in the optical frequency these fringe patterns will shift, the phase shift can therefore be evaluated from the shift in position, $\Delta s$, between the flow on and flow off images:

$$
\left(\Delta \varphi-\Delta \varphi_{0}\right)=2 \pi \frac{\Delta s}{s}
$$

Where $\mathrm{s}$ is the un-shifted fringe spacing, $\Delta s$ is the shift in the fringe position and $\left(\Delta \varphi-\Delta \varphi_{0}\right)$ is the phase shift. The Doppler shift can then be found using equation (6). This processing technique can only calculate the velocity field along profiles corresponding to fringe minima, not across the whole image plane. Seiler et al. [8] have reported a similar system using a Michelson interferometer. However, the MZI based approach can provide double the number of profiles under the same measurement conditions due to the availability of two complementary outputs, compared to the single output available from a Michelson interferometer. Although multiple components of the velocity can be measured using this approach, the calculation of orthogonal velocity components as 
each velocity component is calculated only at fringe minima positions and these are unlikely to coincide when multiple components are mapped together.

\subsection{Calculation of phase using the Normalised intensity}

In practice, the range of the normalised intensity will not be within $-1 \leq I_{N} \leq 1$ across the whole image due to optical distortions in the MZI. To take account of this equation (4) can be rewritten as:

$$
\begin{aligned}
I_{N}(i, j) & =\frac{I_{1}(i, j)-I_{2}(i, j)}{I_{1}(i, j)+I_{2}(i, j)} \\
& =\left(\frac{I_{N \max }(i, j)+I_{N \min }(i, j)}{2}\right)+\left(\frac{I_{N \max }(i, j)-I_{N \min }(i, j)}{2}\right) \cos (\Delta \varphi(i, j))
\end{aligned}
$$

where the indices $(i, j)$ give the pixel position in the images. $I_{N \text { max }}$ and $I_{N \text { min }}$ are the maximum and minimum values for the normalised intensity at each pixel, which can be approximately evaluated from the closest maximum and minimum in the fringe pattern. The phase difference, $\Delta \varphi(i, j)$, for each pixel, can then be found by rearranging equation (8).

$$
\Delta \varphi(i, j)=\arccos \left(\frac{2 \cdot I_{N}(i, j)-\left(I_{N \max }(i, j)+I_{N \min }(i, j)\right)}{I_{N \max }(i, j)-I_{N \min }(i, j)}\right) \pm n \pi \quad n=0,1,2,3 \ldots
$$

This calculation is performed for both the flow on and flow off fringe patterns and the phase shift, $\left(\Delta \varphi-\Delta \varphi_{0}\right)$ calculated. The Doppler shift is calculated as in equation (7).

\subsection{Phase calculation using Fourier domain processing}

A spatial carrier fringe method can be used to evaluate the phase of the recorded fringe patterns [10]. Figure 5 shows an overview of this method for a simulated fringe pattern. Two fringe images are recorded, a flow-off image containing only the carrier fringe phase variation and a flow-on image containing the carrier fringes and an addition phase shift due to the Doppler shift (figure 5, a). The two-dimensional Fourier transformation is calculated for both the flow-on and flow-off fringe images (figure 5, b). One of the side spectra in each of the transformed images is selected and isolated (figure 5, c). The inverse two-dimensional FFT is the calculated, and the phase calculated (figure 5, d) for both the flow-on and flow-off images using equation (10):

$$
\varphi=\tan ^{-1}\left(\frac{\operatorname{Im}(c(x, y))}{\operatorname{Re}(c(x, y))}\right)
$$

The phase shift can then be calculated by subtracting the flow-on phase and the flow-off phases (figure 5, e), finally the Doppler shift can be calculated using equation (7). 
Flow-Off

\section{Recorded fringe} patterns

(a)

(b)

(c)

Isolation

2D FFT

2D inverse FFT

\&

Phase calculation:

(d)
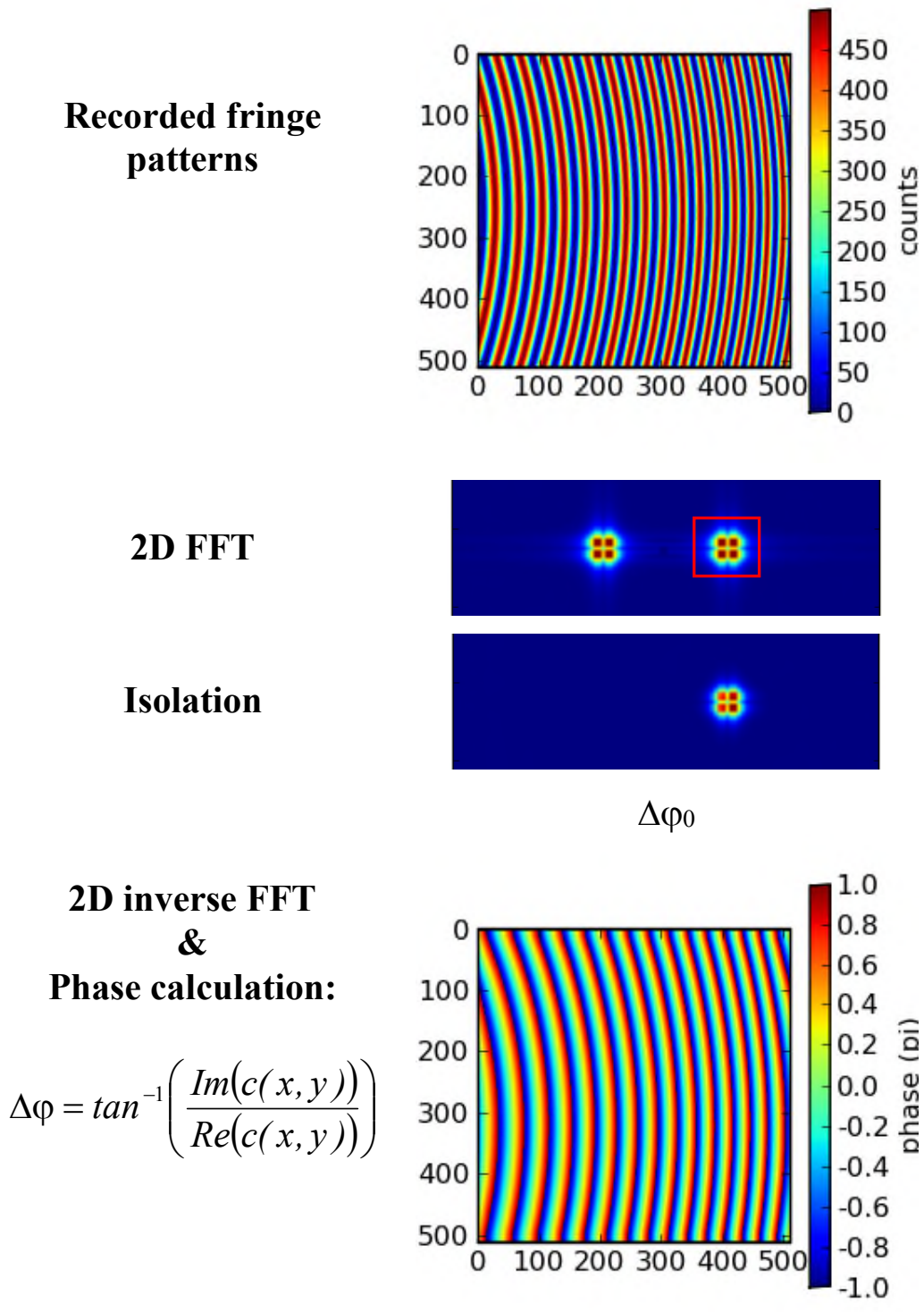

$\Delta \varphi_{0}$
Flow-On

\section{Calculate Phase shift} (left): $\left(\Delta \varphi-\Delta \varphi_{0}\right)$

(e) Calculate Doppler shift

$$
\begin{aligned}
& \text { (right): } \\
& \Delta v=\frac{c}{\Delta l} \frac{\left(\Delta \varphi-\Delta \varphi_{0}\right)}{2 \pi}
\end{aligned}
$$

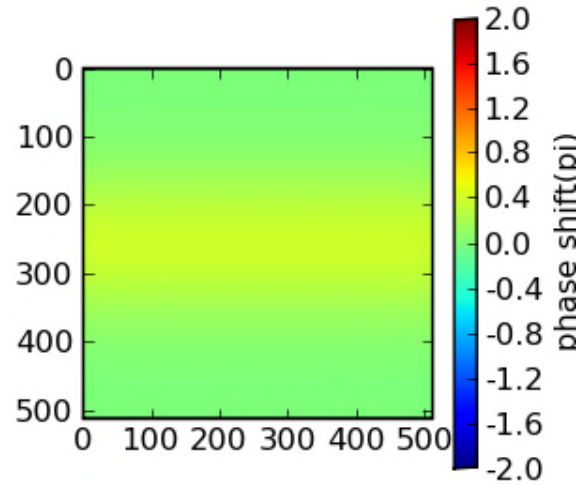

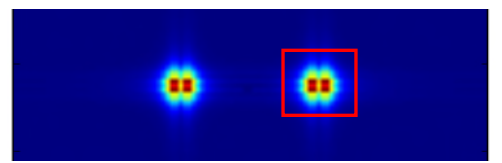

(i⿱一⿻上丨

$\Delta \varphi$
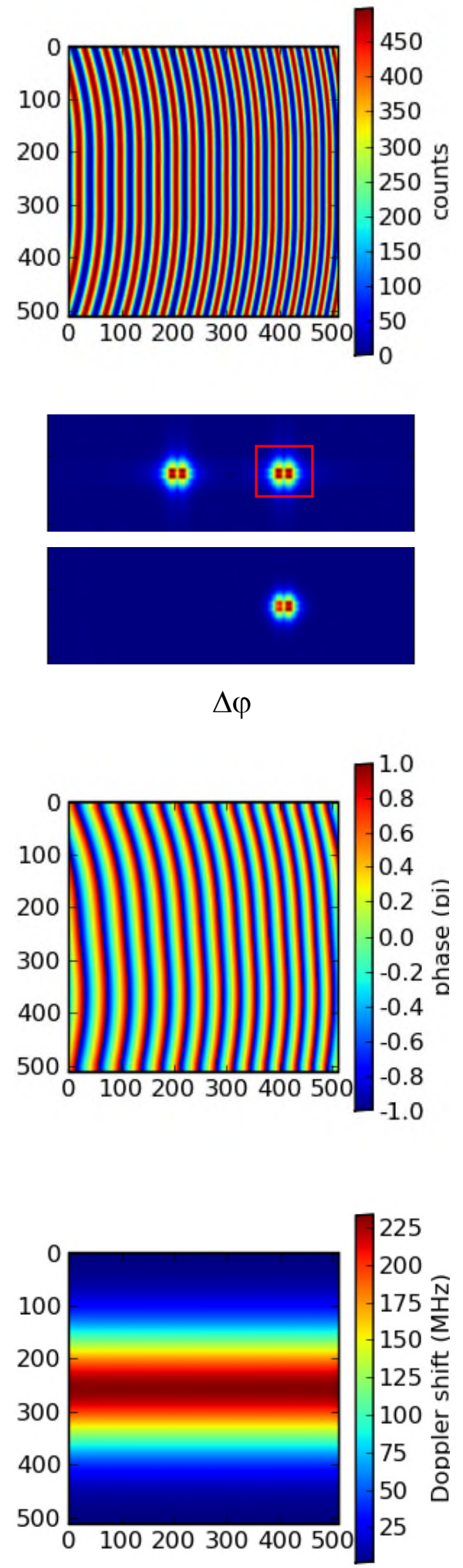

Fig. 5 The two-dimensional phase demodulation scheme using simulated data. (a) Flow-on and flow-off fringe images are recorded. (b) The 2D-FFT of the fringe patterns is calculated. (c)-(d) The phase from each is calculated independently from the isolated side spectrum (e) The phase shift found by subtraction, the Doppler shift can then be found from this phase shift. 


\section{Results}

A rotating disc was used as the first target to validate the 3D MZI-PDV system providing a welldefined velocity field. The rotating disc is about $200 \mathrm{~mm}$ in diameter and coated with white paint in order to enhance the light scattering. The maximum circumferential velocity in the field of view $(\sim 100 \mathrm{x} 100 \mathrm{~mm})$ was $\sim \pm 34 \mathrm{~ms}^{-1}$ at the disc edge that was measured independently using an optical tachometer. Images were captured using a 5 second integration time and processed using the method described in section 4.3. An example of a typical measurement is shown in figure 6(a). This shows a single velocity component using a single arm of the imaging fibre bundles. Figure 6(b) shows the error when the theoretical velocity component is subtracted and figure 6(c) shows the histogram of this error, the standard deviation in the error for all measured components was $<1 \mathrm{~ms}^{-1}$. Figure 7 shows the computed orthogonal velocity components, the in-plane components are represented by the vectors and the out-of-plane by the colour scale.

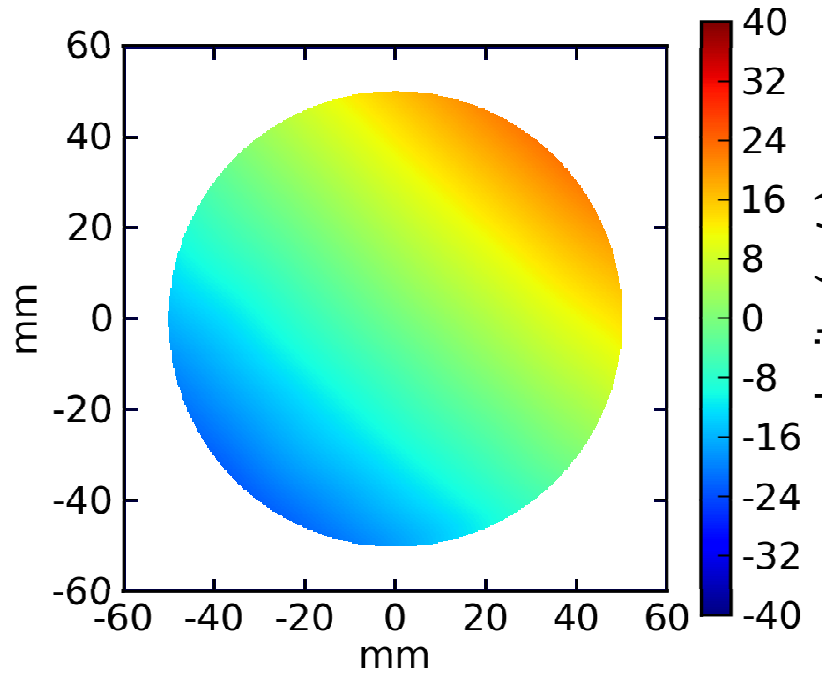

(a)

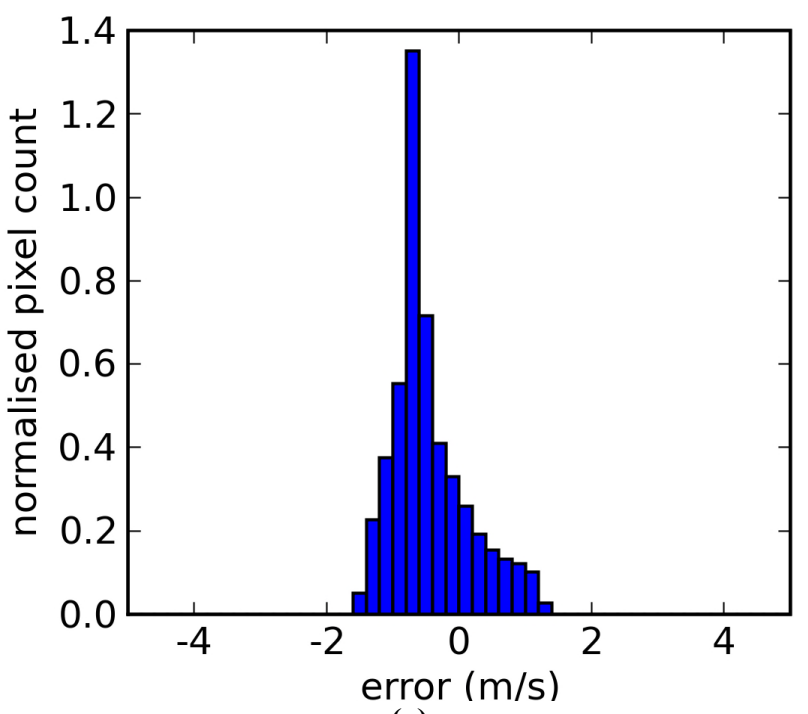

(c)

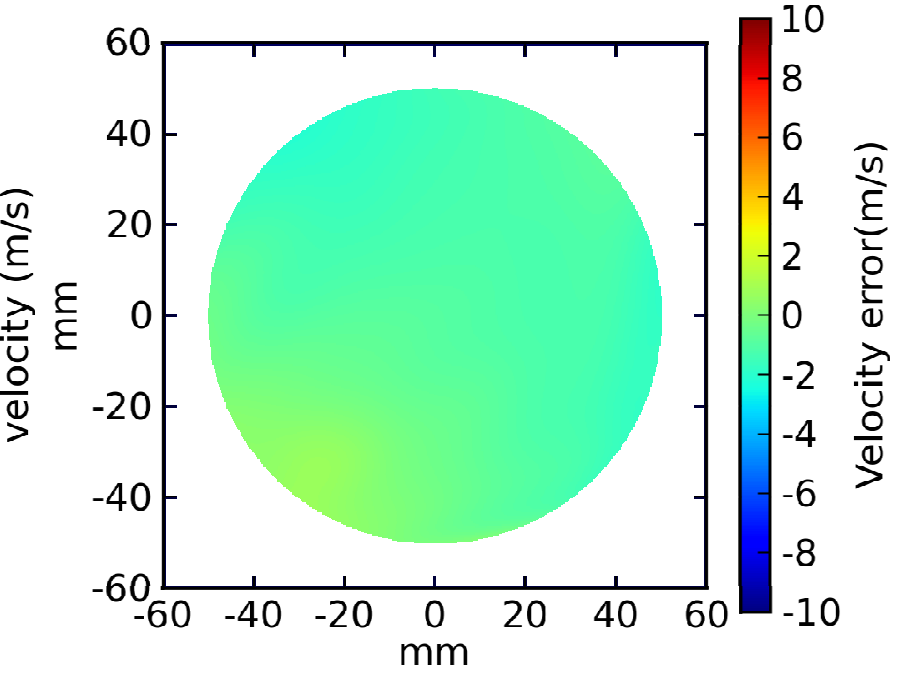

(b)

Fig 6. Example results for a measurement on a rotating disc, calculated using the Fourier domain processing technique, showing (a) a single velocity component, (b) the remaining error when the theoretical component is subtracted (c) a histogram of the remaining error for this component. 


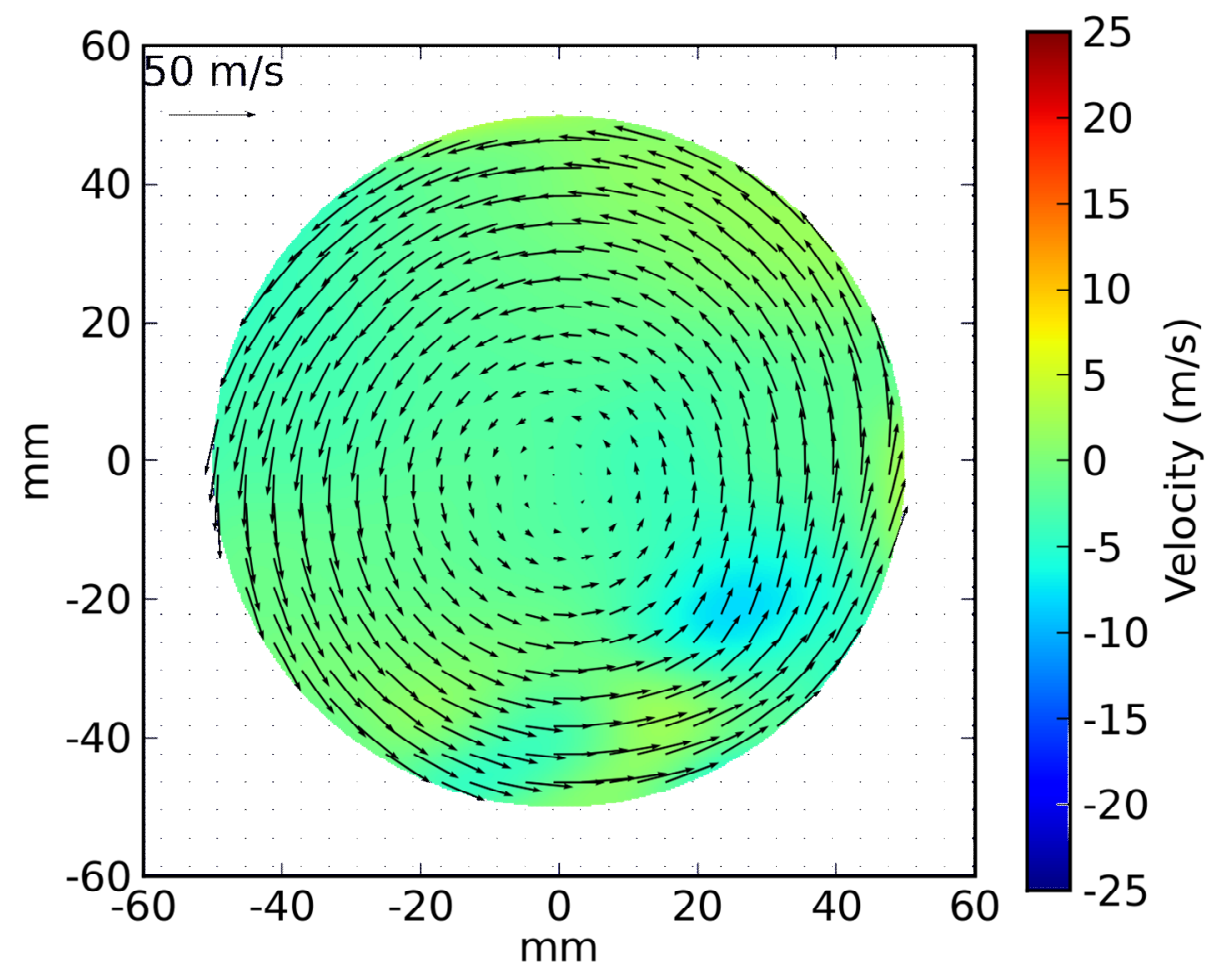

Fig. 7 Computed orthogonal components of the velocity for a measurement on a rotating disc, calculated using the Fourier domain processing technique. In plane components are shown as vectors and the out-of-plane component using the colour scale.

The system was used to make measurements on a seeded air jet, with a $20 \mathrm{~mm}$ diameter smooth contraction nozzle. The exit velocity of the jet was $\sim 80 \mathrm{~ms}^{-1}$. The air intake to the jet, and the surrounding co-flow, were seeded using a Concept Engineering Vicount compact smoke generator producing dense and dry smoke particles in the $0.2-0.3 \mu \mathrm{m}$ diameter range. The observation directions were selected to be in the strong forward scattering so as to ensure sufficient signal on the CCD cameras and images were recorded using an integration time of 10 seconds. The 'flow off' images were obtained using the seeded jet with the jet flow as slow as possible $\left(\sim<1 \mathrm{~ms}^{-1}\right)$, this was necessary as both sides of the light sheet were viewed so it was not possible to use a solid target such as a piece of card illuminated by the light sheet. Figure 8(a) shows a three-component velocity measurement vertically through the centre of the air jet. The in-plane components shown as vectors and the out-of-plane component using the colour scale. This was calculated using the Fourier domain processing technique. A mask was applied to remove regions where a reliable calculation was not possible. These are the outer regions of the jet in which there was insufficient scattered light or towards the edge of the image where there was poor fringe visibility due to the vignetting effect in the Mach-Zehnder interferometer. Figure 8(b) shows profiles through the jet calculated using the normalised intensity method. The blue points show the vertical component of velocity calculated using three observation directions and the red using all four measurement channels. The solid line is a theoretical jet profile calculated using empirical equations, a good agreement between this and the experimental data can be seen. 


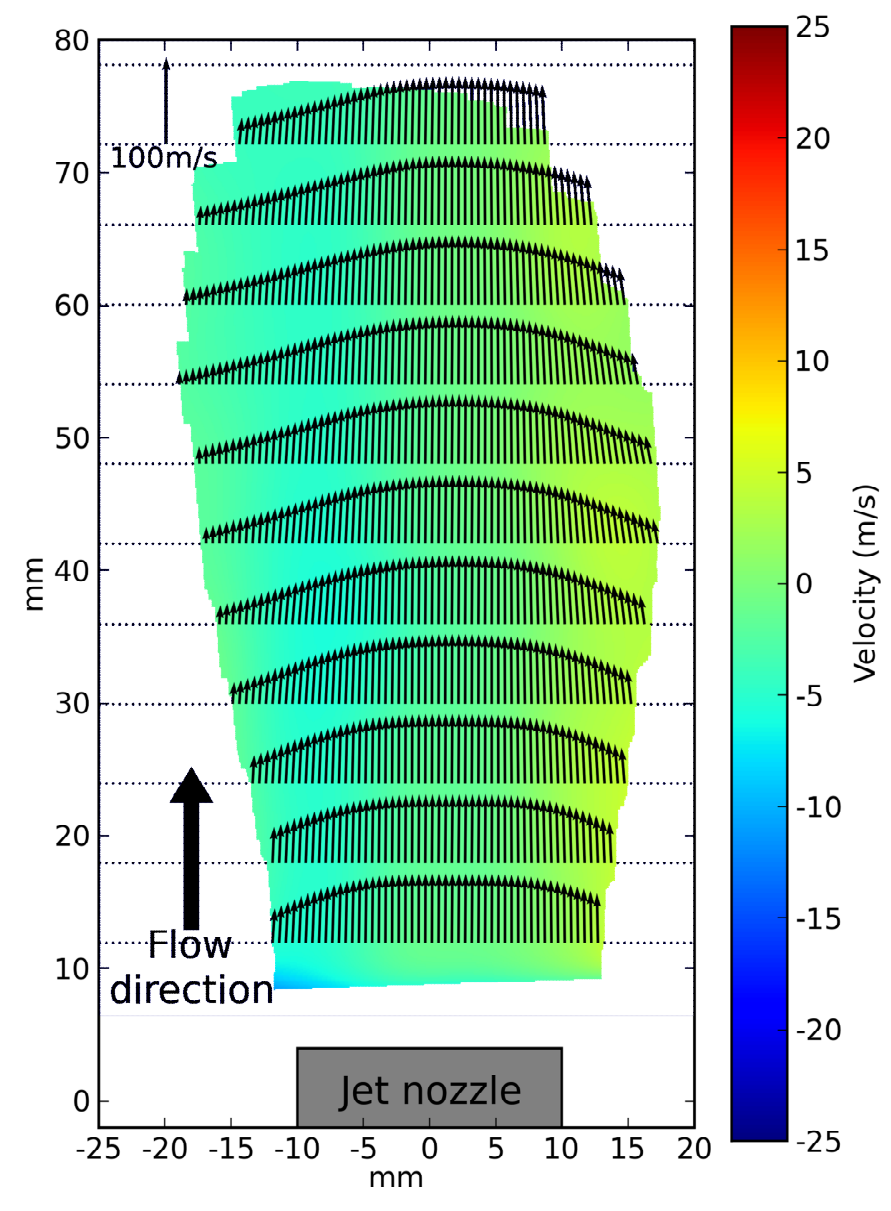

(a)
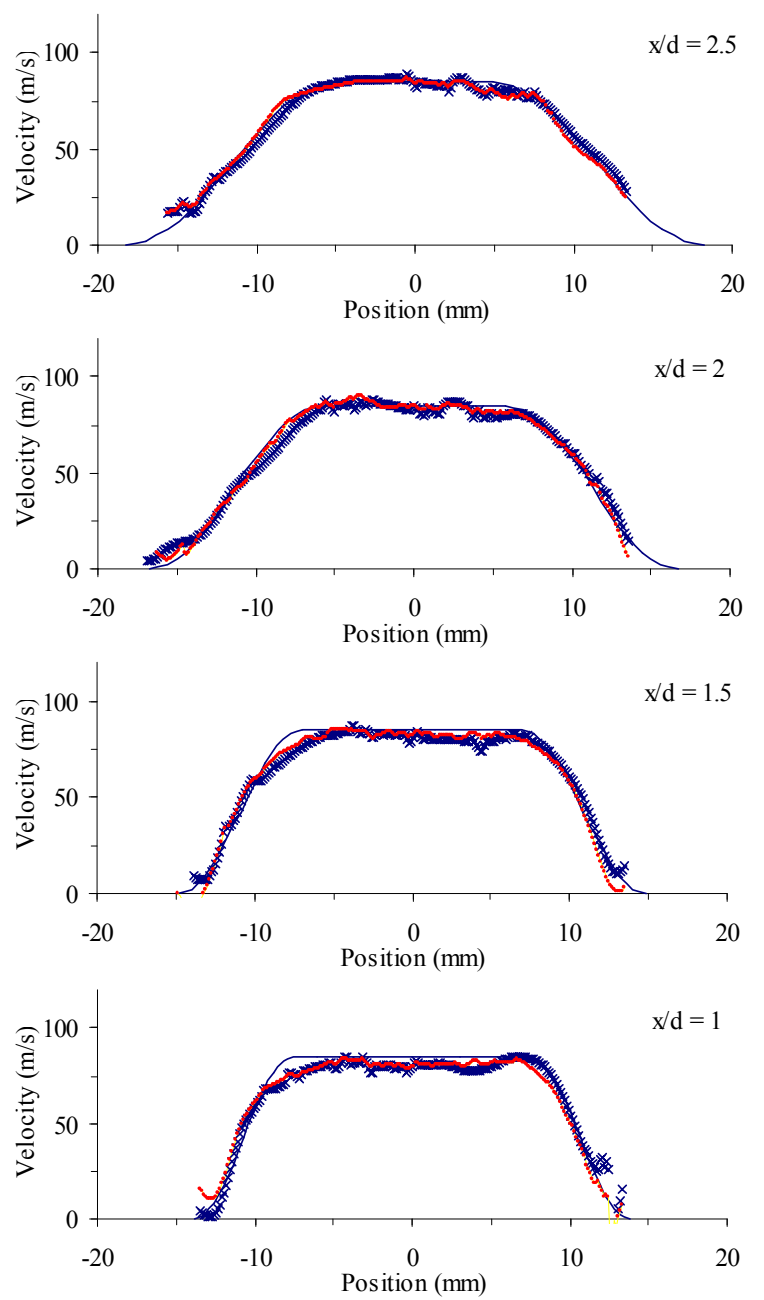

(b)

Fig. 8 Examples of MZI-PDV measurements on a seeded air jet (a) Processed orthogonal velocity components calculated using the Fourier domain processing technique. The in-plane components shown as vectors and out-ofplane using the colour scale. (b) Profiles through the jet at various positions downstream of the nozzle - calculated using the normalised intensity technique. Blue points - calculated using three components, red points calculated using four components.

\section{Conclusions}

A PDV technique using a MZI filter instead of a molecular filter has been demonstrated. A comparison of the two methods is shown in table 1. A validation measurement on a rotating disc was presented with the standard deviation of the error in measured velocity components found to be $\sim 1 \mathrm{~m} / \mathrm{s}$. Measurements on a seeded air jet were made with good agreement to the expected velocities calculated using empirical equations describing the jet.

Three different methods for calculating the interferometer phase shift between images recorded with the flow-on and flow-off have been described. The fringe skeletonization method, described in section 3.1 is relatively simple to implement but has the disadvantage that the shift cannot be evaluated at every pixel but only along fringe minima. Because of this it is not practical to make three component measurements using this method, as measurement positions are unlikely to coincide for different measured velocity components. The methods based upon the normalized intensity and Fourier domain processing both provide velocity measurements at each pixel, with the Fourier transform method working most effectively. 
Table 1 A general comparison of Iodine molecular filters and interferometers for PDV

\begin{tabular}{|c|c|c|}
\hline & Molecular filter & Interferometric filter \\
\hline Principle & Gas absorption & Optical interference \\
\hline Transfer function & Fixed to absorption line & Co-sinusoidal form \\
\hline Stability & Temperature sensitive & $\begin{array}{l}\text { Temperature and vibration } \\
\text { sensitive }\end{array}$ \\
\hline Light source & $\begin{array}{l}\text { Limited to those able to be tuned to } \\
\text { an absorption line. }\end{array}$ & $\begin{array}{c}\text { Any laser of sufficient power and } \\
\text { narrow linewidth }\end{array}$ \\
\hline $\begin{array}{l}\text { Velocity range / } \\
\text { resolution }\end{array}$ & $\begin{array}{l}\text { Fixed to absorption line shape } \\
\text { (some control from vapour } \\
\text { pressure, thermal and pressure } \\
\text { broadening) }\end{array}$ & $\begin{array}{c}\text { Can be tuned to select velocity } \\
\text { range and resolution }\end{array}$ \\
\hline Complexity & Simple & Complex structure \\
\hline Availability & Commercial (limited) & Custom designed \\
\hline
\end{tabular}

\section{Acknowledgements}

Zenghai Lu acknowledges an Overseas Research Scholarship (ORS) award from the Committee of Vice Chancellors and Principals, UK. This work was also funded by an Engineering and Physical Sciences Research Council (EPSRC) platform grant.

\section{References}

1. Elliott G. S., and Beutner T. J., "Molecular filter based planar Doppler velocimetry", Progress in Aerospace Sciences, 35(8), 799-845 (1999).

2. Nobes D.S., Ford H.D. and Tatam R.P., "Instantaneous, three-component planar Doppler velocimetry using imaging fibre bundles", Experiments in Fluids, 36(1), 3-10 (2004).

3. Meyers J.F. and Komine H., "Doppler Global Velocimetry: a new way to look at velocity", Laser Anemometry, 1, 273-277 (1991).

4. Reinath M.S. "Doppler Global Velocimeter Development for the Large Wind Tunnels at Ames Research Center", NASA Technical Memorandum, 112210 (1997).

5. Seiler F, Havermann M, George A, Leopold F and Srulijes J. "Planar velocity visualization in high-speed wedge flow using Doppler picture velocimetry (DPV) compared with particle image velocimetry (PIV)", Journal of Visualization 6 253-62 (2003).

6. Seiler F and George A, "Doppler picture velocimetry (DPV) applied to high-speed shock tunnel flows", 11th Int. Symp. Flow Visualization, (Notre Dame/IN, USA), (2004).

7. Lu Z.H., Charrett T.O.H., Ford H.D. and Tatam R.P., "Mach-Zehnder interferometric filter based planar Doppler velocimetry (MZI-PDV)", Journal of Optics A: Pure and Applied Optics, 9, 1002-13, (2007).

8. Wernert P., Martinez B., George A., Leopold F. and Seiler F., "Development of Doppler based planar velocimetry techniques for high-speed flows at ISL", 8th Int. Symp. Fluid Control, Measurement and Visualization (Chendu, CN) Paper 316, (2005).

9. Charrett T.O.H., Nobes D.S., and Tatam R.P., "Investigation into the selection of viewing configurations for three component planar Doppler velocimetry (PDV) measurements.", Applied Optics. 46, 19, pp 4102-4116, (2007).

10. Robinson D.W. and Reid G.T., "Interferogram analysis: digital fringe pattern measurement techniques", Institute of Physics publishing, Bristol (1993). 
2008

Mach-Zehnder interferometric filter based planar Doppler velocimetry (MZI-PDV)

Lu, Zenghai $\mathrm{H}$.

Springer

pÿLu ZH, Charrett TOH, Ford HD, Tatam RP, Mach Zehnder interferometric filter based planar Doppler velocimetry (MZI-PDV), Proceedings of 14th International Symposium on Applications of Laser Techniques to Fluid Mechanics, 7-10 July 2008, Lisbon, Portugal https://dspace.lib.cranfield.ac.uk/handle/1826/12453

Downloaded from Cranfield Library Services E-Repository 